\title{
Flow Cytometric Assessment of AKT Signaling in Platelet Activation: An Alternative Diagnostic Tool for Small Volumes of Blood
}

\author{
K. Althaus ${ }^{1,2} \quad$ M. Wagner ${ }^{1} \quad$ I. Marini $^{1} \quad$ T. Bakchoul $\left.\right|^{1,2} \quad$ L. Pelzl ${ }^{1}$ \\ ${ }^{1}$ Transfusion Medicine, Medical Faculty of Tuebingen, University \\ Hospital of Tuebingen, Tuebingen, Germany \\ ${ }^{2}$ Centre for Clinical Transfusion Medicine, University Hospital of \\ Tuebingen, Tuebingen, Germany \\ Address for correspondence Karina Althaus, MD, Center for Clinical \\ Transfusion Medicine, University Hospital of Tuebingen, Otfried- \\ Mueller-Straße 4/1, 72076 Tuebingen, Germany (e-mail: karina. \\ althaus@med.uni-tuebingen.de).
}

Hämostaseologie 2020;40(Suppl 1):S21-S25.

\begin{abstract}
Keywords

- AKT

- platelet

- acquired platelet disorders

- inherited platelet disorders

- platelet function

Introduction The diagnosis of platelet function disorder in children is challenging. Light transmission aggregometry is the gold standard for platelet function disorders. However, large blood volumes are required. Currently, there are no existing tools for the diagnosis of platelet function disorders that use small blood volumes. AKT signaling plays a central role in platelet activation during hemostasis and might be visualized by flow cytometry.

Methods Platelet-rich plasma obtained by centrifugation of citrated blood from healthy volunteers was activated with arachidonic acid, thrombin receptor activating peptide-6 (TRAP-6), collagen, adenosine diphosphate ADP, collagen-related peptide (CRP), and epinephrine. After platelet activation, the phosphorylation of AKT was assessed by flow cytometer using a Navios cytometer.

Results Healthy volunteers showed a reproducible phosphorylation of AKT upon activation. In comparison to nonactivated platelets, we documented an increase in PAKT expression with all agonists. Especially TRAP-6 and CRP caused considerable increase in percentage of PAKT expression throughout all the tested healthy volunteers.

Conclusion An activation of the AKT-signal pathway by different agonists can clearly be detected on the flow cytometer, indicating that the visualization of signaling in platelets by flow cytometry might be an efficient alternative for light transmission aggregometry to test platelet function in children.
\end{abstract}

\section{Introduction}

Platelets play a key role in primary hemostasis and their activation as well as aggregation is mediated by a complex interaction involving distinct signal transduction pathways. The most important signaling molecules involved in platelet activation are phospholipase C (PLC), protein kinase C (PKC), and phosphatidylinositide-3-kinase (PI3K). ${ }^{1}$ Platelet agonists like thrombin, adenosine diphosphate (ADP), thromboxane A2

received

September 11, 2020

accepted

October 5, 2020

(TXA2), and epinephrine activate more than one of these molecules. The only exception is collagen which activates PLC via tyrosine kinase directly. ${ }^{2}$ The activation of PLC, PKC, and PI3K results in granule release and glycoprotein (GP) IIbIIIa activation and aggregation. The serine/threonine kinase AKT is a crucial player as a downstream effector of PI3K in platelets. There are three different isoforms in platelets. In fact, studies with $\mathrm{AKT}^{-1-}$ and $\mathrm{AKT}^{-1-}$ knockout mice showed that

(c) 2020 Georg Thieme Verlag KG Stuttgart · New York
DOI https://doi.org/

10.1055/a-1282-1989. ISSN 0720-9355. 
normal platelet activation is not possible without AKT1. ${ }^{3-5}$ AKT2 isoform is the most predominantly expressed isoform in the human platelets, but AKT1 and AKT3 are important for thrombus formation, secretion, and aggregation as well. ${ }^{6}$

Platelet function disorders are a common cause for diseases of hemostasis. Platelet function disorders can be congenital or acquired. Clinical manifestations include hematoma, epistaxis, severe bleeding episodes after injuries or surgery, and menorrhagia in female patients. Unfortunately, the diagnosis of platelet function disorder in children is still challenging. Although light transmission aggregometry is the gold standard for platelet function disorders, large blood volumes are required. Currently, there are no existing tools for the diagnosis of signal transduction defects in small blood volumes. Also in drug monitoring, a sufficient platelet function testing for resistance in patients or to change therapy on the basis of such tests for effectiveness of inhibition is lacking. ${ }^{7}$ In this study, we present a flow cytometry (FC)based activation assay with measurement of the serine/threonine kinases AKT, which is involved in complete activation process of platelets. ${ }^{3-5}$

\section{Methods}

\section{Blood Samples}

Citrated blood samples were collected from healthy volunteers after obtaining written consensus. Inclusion and exclusion criteria were no intake of drugs within the last 10 days. Blood was centrifuged within 1 hour after collection at $120 \mathrm{~g}$ for 15 minutes at room temperature (RT) without break in order to obtain platelet-rich plasma (PRP).

\section{Cell Activation}

PRP was incubated with ADP $(10 \mu \mathrm{M}$; Hart Biologicals, Hartlepool, UK), TRAP-6 (10 $\mu \mathrm{M}$; Hart Biologicals), collagen $(10 \mu \mathrm{g} / \mathrm{mL}$; Crono-Par, Harvertown, Pennsylvania, United States), arachidonic acid (AA; $1.5 \mathrm{mM}$; Hart Biologicals), and $10 \mu \mathrm{M}$ epinephrine (Hart Biologicals) at $37^{\circ} \mathrm{C}$ for 15 minutes at RT. Next, cells were stained with P-selectin ([CD62P] 1:20, CLBThromb/6, Beckman Coulter) for 30 minutes at RT in the dark. Finally, after one washing step (650 $g, 7$ minutes, RT) with PBS (Biochrom GmbH, Berlin, Germany), the CD62P expression was determined by FC (Navios, Beckman-Coulter, USA). Test results were calculated as fold increase (FI) compared to resting cells.

\section{Signaling with and without Activation}

Signal transduction pathways were assessed upon incubation with $1.5 \mathrm{mM}$ AA, $20 \mu \mathrm{M}$ TRAP-6, $10 \mu \mathrm{g} / \mathrm{mL}$ collagen, $10 \mu \mathrm{M}$ ADP, $5 \mu \mathrm{g} / \mathrm{mL}$ collagen-related peptide (CRP; Hart Biologicals), and $15 \mu \mathrm{M}$ epinephrine at $37^{\circ} \mathrm{C}$ for 5 minutes. The commercially available PerFIX-kit (Beckmann Coulter, Marseille, France) was used to fix and permeabilize the cells according to the manufacturer's instructions as previously described. ${ }^{8}$

Next, the cells were characterized using triple staining with antibodies against CD41-PC5 (Beckmann Coulter), AKT1-APC (Invitrogen, Eugene, Oregon, United States), and pAKT1-PE (Invitrogen, Eugene). The samples were then analyzed by FC. Platelets were identified according to their size and granularity
(FSC: forward scatter and SSC: side scatter) as well as the expression of the specific CD41-PC5 marker.

A total volume of $140 \mu \mathrm{L}$ PRP $(250,000$ cells $/ \mu \mathrm{L})$ was required for these analyses.

\section{Western Blot Analysis}

Protein levels of AKT1 and pAKT1 were determined by Western blot. After isolation of platelets from healthy donors, platelets were activated by $5 \mu \mathrm{g} / \mathrm{mL}$ CRP and centrifuged for 5 minutes, $700 \mathrm{~g}$ at $4^{\circ} \mathrm{C}$. Later on, the pellet was washed with ice-cold PBS and suspended in 50- $\mu$ L ice-cold RIPA lysis buffer (ThermoFisher Scientific, Paisley, UK) containing HALTTM protease and phosphatase inhibitor-cocktail (ThermoFisher Scientific). Protein concentration was determined using the NanoDrop One (VWR, Bruchsal, Germany). For 10 minutes, $250 \mu$ of protein was solubilized in fluorescent-compatible sample buffer (Invitrogen, Carlsbad, California, United States) at $95^{\circ} \mathrm{C}$. The proteins were separated by electrophoresis for 60 to 90 minutes using $12 \%$ SDS-PAGE gels in glycine-tris buffer. Thereafter, probes were transferred to polyvinylidene difluoride (PVDF) membranes $(0.45 \mu \mathrm{m}$; Merck, Tullagreen, Ireland). After blocking with $5 \%$ milk in TBS-T buffer $(20 \mathrm{mM}$ Tris, $140 \mathrm{mM} \mathrm{NaCl}, 0.1 \%$ Tween, $\mathrm{pH}$ 7.6) at RT for 1 hour, the membranes were incubated with primary anti-AKT1 (Invitrogen, Eugene) and pAKT1 (Invitrogen, Eugene) and antimouse GAPDH (1:1,000; Cell Signaling, Danvers, Massachusetts, United States) at $4^{\circ} \mathrm{C}$ overnight. After washing with TBS-T buffer, the membranes were incubated with the appropriate secondary antirabbit antibody or with a secondary antimouse antibody conjugated with IRDye680/IRDye800 (1:3,000; LI-COR, Lincoln, Nebraska, United States) for 1 hour at RT. Protein bands were detected after additional washes (TBST) with an imaging system (LI-COR). Western blots were analyzed by ImageJ software (NIH, Bethesda, United States). The results are shown as the ratio of total AKT1 and pAKT1 to GAPDH.

\section{Statistical Analysis}

The statistical analysis was performed using GraphPad Prism, Version 7.0 (GraphPad, San Diego, California, United States). Nonparametric tests were used when data failed to follow a normal distribution as assessed by the D'Agostino and Pearson omnibus normality test. Group comparison was performed using the Wilcoxon rank-sum test. All analyses were two-tailed and a $p$-value of $<0.05$ was defined to indicate a statistically significant difference.

\section{Results}

To assess the activation status of platelets, the expression of CD62P was estimated in the absence (baseline) and presence of ADP, TRAP-6, AA, collagen, and epinephrine. Higher FI values for all activation markers were observed after activation ( - Fig. 1) with highest FI values upon induction with AA (mean FI: 6.85 [range: 5.61-8.81], $p=0.0001$ ), whereas the lowest FI value (mean FI: 1.11 [range: $0.99-1.21$ ], $p=0.0019$ ) was observed after induction with epinephrine.

Healthy volunteers showed a reproducible phosphorylation of AKT. In the resting condition, we detected only AKT-kinase expression but no pAKT ( - Fig. $2 \mathrm{~A}-\mathrm{C}$ ). In comparison to 


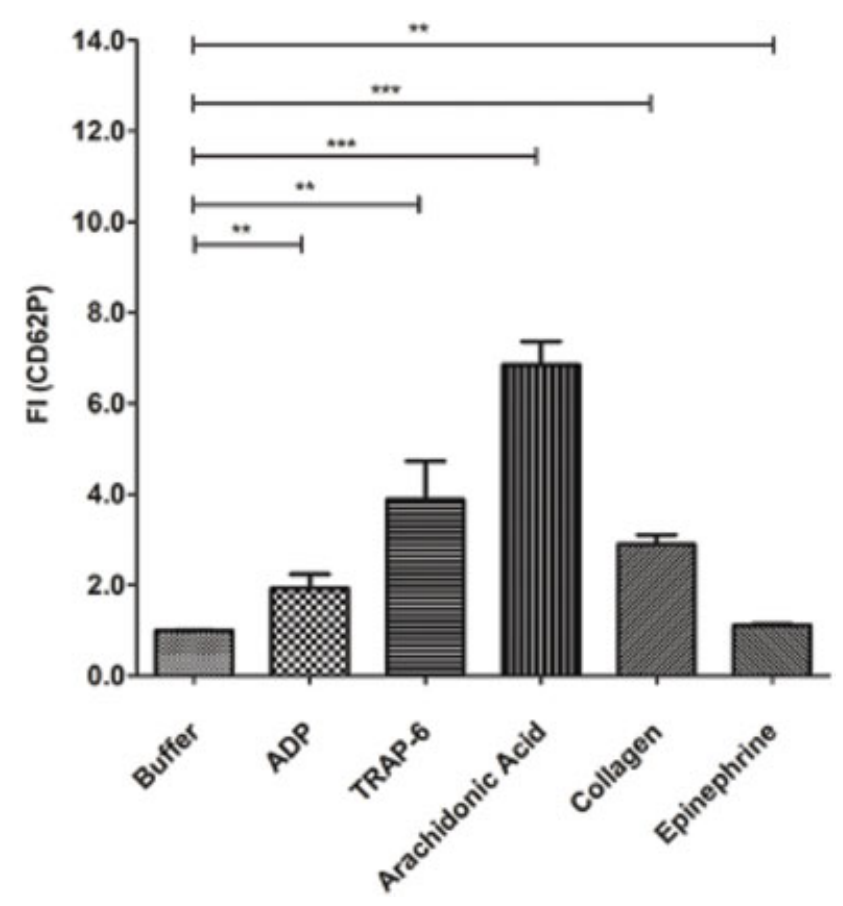

Fig. 1 Expression of P-selectin. The expression of P-selectin (CD62P) upon stimulation with adenosine diphosphate (ADP), thrombin receptor activating peptide-6 (TRAP-6), collagen, arachidonic acid, and epinephrine was assessed. Data are presented as mean \pm SEM of the measured fold increase $(\mathrm{FI})$ compared to resting cells. ns, not significant, ${ }^{*} p<0.05,{ }^{* *} p<0.01,{ }^{* * *} p<0.001$, and ${ }^{* * * *} p<0.0001$. nonactivated platelets, we documented, calculating the mean FI, an increase in pAKT expression with all agonists ( - Fig. 2DF; - Fig. 3). We confirmed these data by the Western blot analysis. We found AKT in resting and activating platelets, while pAKT was detected in activated platelets only ( - Fig. 4). Lowest increases in FC were reached by activation with epinephrine (mean: 3.21 [range: 1.10-6.42]). Moderate increase of pAKTwas measured after activation with AA (mean FI: 13.88 [range: 3.96-18.14]) and collagen (mean FI: 10.40 [range: 1.78-20.12]). However, ADP (mean FI: 24.96 [range: 3.62-56.55]), CRP (mean FI: 21.44 [range: 13.11-35.68]), and TRAP-6 (mean FI: 33.00 [range: 13.41-51.62]) induced the highest increases in percentage of pAKT expression throughout all the tested healthy volunteers (-Table 1, -Fig. 3). Comparing the enhanced expression of pAKT and AKT upon incubation with different agonists with resting control cells (PBS), a similar increase in the percentage of double positive cells ( \pm standard error mean) in all samples was observed. In particular, TRAP- 6 versus PBS ( 12.0 vs. $0.4, p<0.001)$; ADP versus PBS (9.6 vs. $0.4, p=0.0002$ ), and CRP versus PBS (12.1 vs. $0.4, p=0.0006$ ) showed the highest increase in comparison to PBS. Moreover, AA versus PBS (6.8 vs. 0.4, $p=0.0005)$ and collagen versus PBS ( 4.0 vs. $0.4, p=0.0054$ ) induced moderate mean percentage increase after stimulation. Epinephrine versus PBS (1.8 vs. $0.4, p=0.0439$ ) resulted in nearly no increase (-Fig. 3B).

\section{Discussion}

This study investigates the feasibility of FC-based assay to investigate the platelet function by visualization of AKT
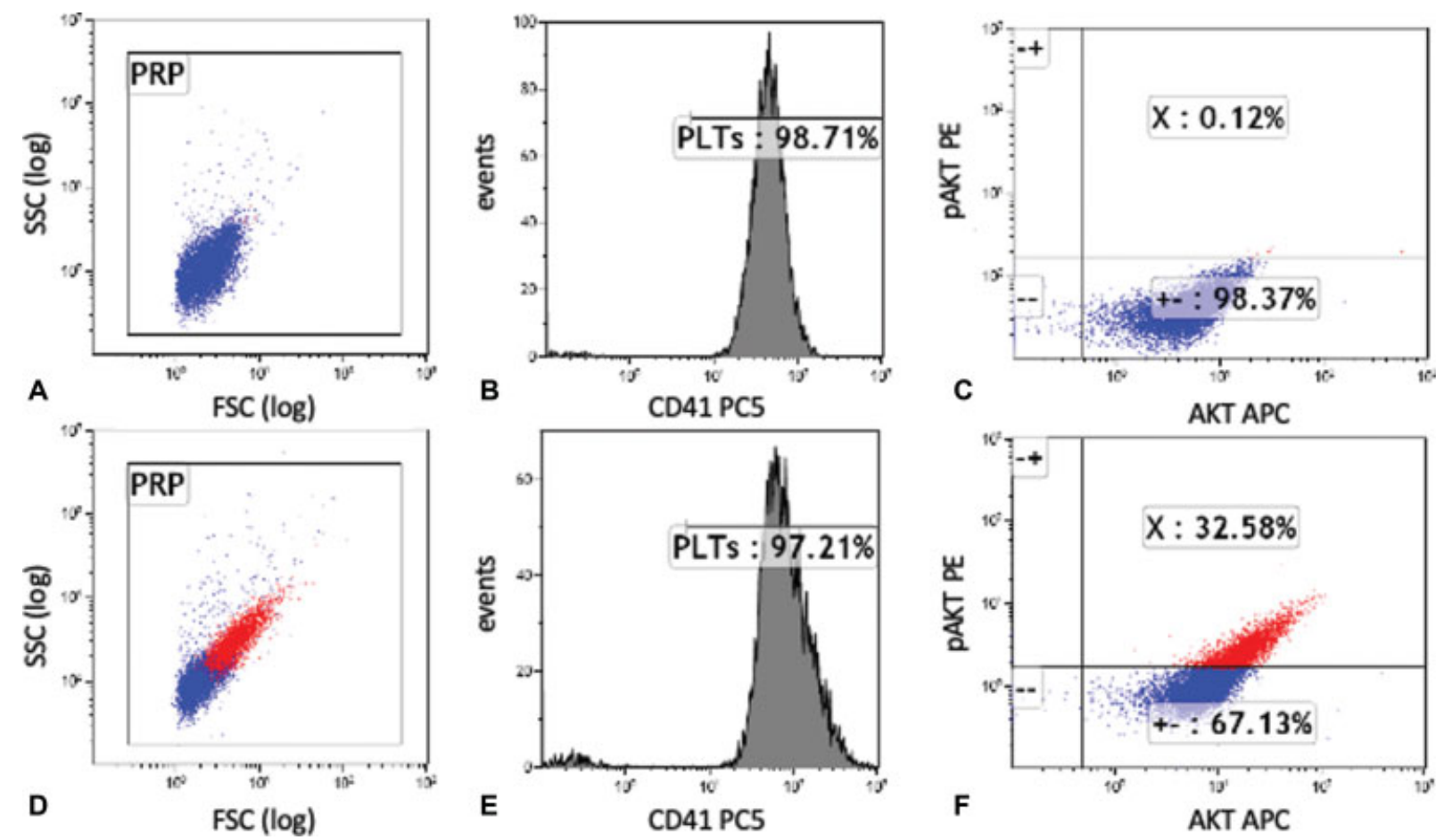

Fig. 2 Representative flow cytometry analysis of AKT/PAKT expression. Platelets (PLTs) were identified according to their size and granularity (FSC: forward scatter and SSC: side scatter) (A, D) as well as the expression of the specific CD41-PC5 marker (B, E). In resting condition, only the kinase AKT was expressed (C). However, upon incubation with the agonist CRP, 33\% of the cells expressed phosphorylated AKT (pAKT), as indicated by the increased of the mean fluorescence intensity $(F)$. 

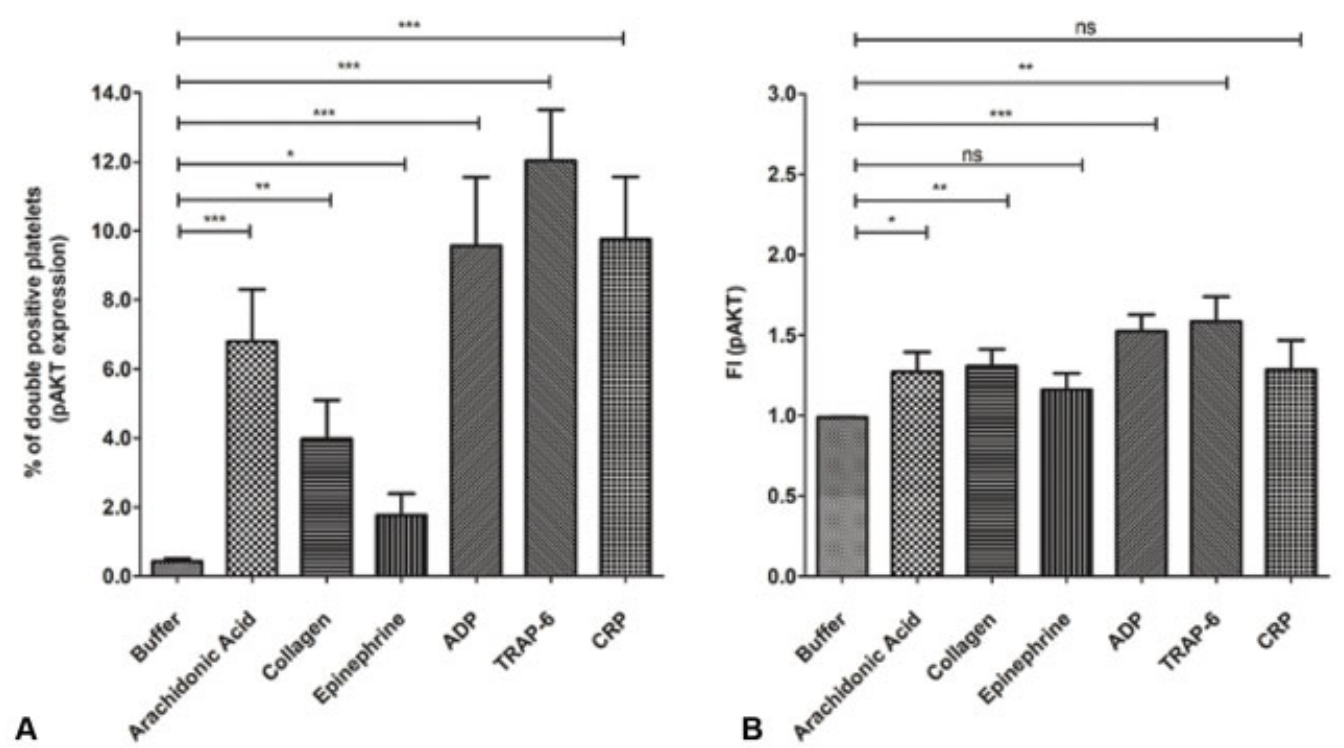

Fig. 3 Phosphorylation of AKT is increased upon platelet's activation. (A) A significant increase of the percentage of AKT-phosphorylated cells upon incubation with the agonists arachidonic acid, collagen, ADP, and TRAP was observed $(n=10)$. (B) Enhanced expression of pAKT and AKT upon incubation with different agonists in comparison to resting control cells (PBS) was detected $(n=10)$. Data are presented as mean \pm SEM. Fl, fold increase; ns, not significant; ${ }^{*} p<0.05,{ }^{* *} p<0.01,{ }^{* * *} p<0.001$, and ${ }^{* * * *} p<0.0001$.
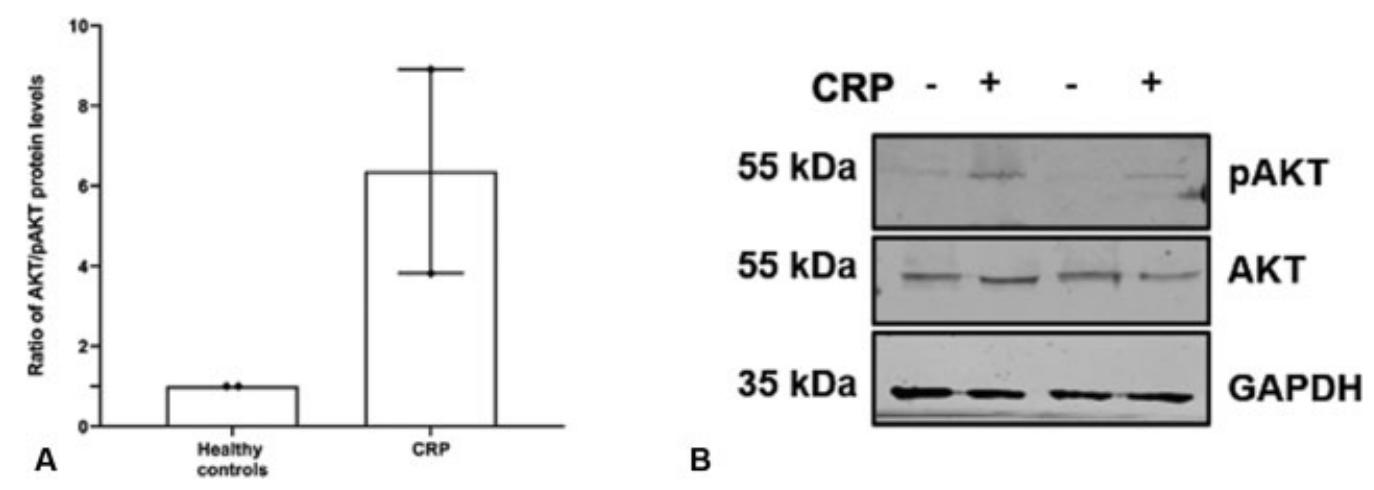

Fig. 4 Western blot analysis of AKT and PAKT in resting and activated platelets: (A) Quantification of AKT and pAKT level expression resting platelets and activated platelets with collagen-related peptide (CRP). (B) Representative original Western blot of AKT, PAKT, and GAPDH.

Table 1 AKT phosphorylation in platelets after activation by different agonists

\begin{tabular}{|l|l|l|l|}
\hline & MV (FI) & Min (FI) & Max (FI) \\
\hline Arachidonic acid $(1.5 \mathrm{mM})$ & 13.88 & 3.96 & 18.14 \\
\hline Collagen $(10 \mu \mathrm{g} / \mathrm{mL})$ & 10.40 & 1.78 & 20.12 \\
\hline Epinephrine $(15 \mu \mathrm{M})$ & 3.21 & 1.10 & 6.42 \\
\hline ADP $(10 \mu \mathrm{M})$ & 2.96 & 3.62 & 56.55 \\
\hline TRAP-6 $(20 \mu \mathrm{M})$ & 33.00 & 13.41 & 51.62 \\
\hline CRP $(5 \mu \mathrm{g} / \mathrm{mL})$ & 21.44 & 13.11 & 35.68 \\
\hline
\end{tabular}

Abbreviations: ADP, adenosine diphosphate; CRP, collagen-related peptide; FI, fold increase; MV, mean value; TRAP-6, thrombin receptor activating peptide.

signaling. We confirmed that the AKT signaling pathway is able to detect the agonist-induced platelet aggregation ${ }^{9}$ and might be a suitable alternative for platelet function testing in children.
FC-based method has the additional advantage of low blood volume needed; for example, it can be performed even in patients with thrombocytopenia. ${ }^{10}$ In addition, compared to light transmission aggregometry, fixed and permeabilized blood samples can be stored overnight and assays can be performed later during routine assessment. With a total volume of $140 \mu \mathrm{L}$, it allows a functional testing and might be combined with morphological studies such as immunofluorescence microscopy to rule out platelet function defects in small children. ${ }^{11}$

A second application of this test system might be in patients taking antiplatelet drugs or for risk assessment in cardiovascular diseases. As PI3K inhibitors became more and more important as a therapeutic option in thrombosis and cardiovascular disease, ${ }^{12}$ this method might be a diagnostic option for drug monitoring or risk stratification in cardiovascular diseases. ${ }^{13}$ Detection of these slight defects in signaling might be helpful in the prediction of bleeding under antiplatelet therapy. 
Our study showed that pAKT detection using FC might not be suitable for agonists like epinephrine. Therefore, further kinase pathways like SYK or SRC should be assessed in the future.

In conclusion, visualization of AKT signaling in platelets by FC might be an alternative for light transmission aggregometry in young children. It should also be emphasized that it is not clear whether the AKT pathway described here is equivalent to the light transmission aggregometry. But it is a practicable option to visualize defects in platelet function and activation by different agonists can be clearly detected using the flow cytometer.

\section{Authors' Contribution}

K.A., M.W., and T.B. designed the study; M.W., L.P., and I.M. performed the experiments. L.P., M.W., T.B. and K.A. collected and analyzed the data and wrote the manuscript. All authors read and approved the manuscript.

\section{Conflict of Interest}

The authors declare no competing financial interests.

\section{Acknowledgments}

This work was supported by grants from TÜFF-Gleichstellungsförderung to K.A. (2563-0-0). This study is part of the doctoral thesis of M. Wagner. We thank Flavianna Rigoni and Karoline Weich for their excellent technical support.

\section{References}

1 Bye AP, Unsworth AJ, Gibbins JM. Platelet signaling: a complex interplay between inhibitory and activatory networks. J Thromb Haemost 2016;14(05):918-930
2 Shattil SJ, Kim C, Ginsberg MH. The final steps of integrin activation: the end game. Nat Rev Mol Cell Biol 2010;11(04):288-300

3 Chen J, De S, Damron DS, Chen WS, Hay N, Byzova TV. Impaired platelet responses to thrombin and collagen in AKT-1-deficient mice. Blood 2004;104(06):1703-1710

4 Woulfe D, Jiang H, Morgans A, Monks R, Birnbaum M, Brass LF. Defects in secretion, aggregation, and thrombus formation in platelets from mice lacking Akt2. J Clin Invest 2004;113(03): 441-450

5 O'Brien KA, Stojanovic-Terpo A, Hay N, Du X. An important role for Akt3 in platelet activation and thrombosis. Blood 2011;118(15): 4215-4223

6 Stalker TJ, Newman DK, Ma P, Wannemacher KM, Brass LF. Platelet signaling. Handb Exp Pharmacol 2012;(210):59-85

7 Michelson AD. Platelet function testing in cardiovascular diseases. Hematology 2005;10(Suppl 1):132-137

8 Gharehbaghian A, Salimian M, Taherian AA, et al. Variations in intraplatelet phospho-VASP expression due to pre-analytical sample preparations, illustration of a quality control issue in platelet pharmacology. Iran J Pharm Res 2015;14(01):321-328

9 Wang $\mathrm{H}$, Ye Y, Wan W, et al. Xinmailong modulates platelet function and inhibits thrombus formation via the platelet

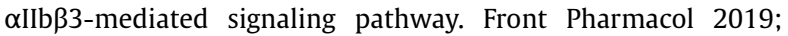
10:923

10 Michelson AD. Evaluation of platelet function by flow cytometry. Pathophysiol Haemost Thromb 2006;35(1-2):67-82

11 Greinacher A, Pecci A, Kunishima S, et al. Diagnosis of inherited platelet disorders on a blood smear: a tool to facilitate worldwide diagnosis of platelet disorders. J Thromb Haemost 2017;15(07): 1511-1521

12 Durrant TN, Hers I. PI3K inhibitors in thrombosis and cardiovascular disease. Clin Transl Med 2020;9(01):8

13 Stojanovic A, Marjanovic JA, Brovkovych VM, et al. A phosphoinositide 3-kinase-AKT-nitric oxide-cGMP signaling pathway in stimulating platelet secretion and aggregation. J Biol Chem 2006; 281(24):16333-16339 